\title{
Insidious abdominal pain? consider spigelian hernia. A case report
}

\author{
Shiva Seetahal* \\ Department of Surgery, Heart of Florida Regional Medical Center (HOFRMC), Davenport, Florida, USA
}

\begin{abstract}
Spigelian hernias are uncommon and represent only $0.1 \%$ of all abdominal hernias. However, by their nature they often present a diagnostic challenge to clinicians. Symptoms for non-obstructed hernias can be vague and non-specific. We discuss a case that highlights some of the diagnostic challenges associated with Spigelian hernias and discuss a simple laparoscopic approach to repair.
\end{abstract}

\section{Introduction}

Spigelian hernias are uncommon and represent only $0.1 \%$ of all abdominal hernias. The clinical features are variable and very often they are diagnosed incidentally during abdominal exploration for persistent abdominal pain. This case report reflects some of these diagnostic challenges as well as a simple repair technique.

\section{Case Report}

A 46 year female presented with the complaint of right lower abdominal and inguinal pain for over 12 months. She described the pain as a sharp and fleeting one that was made worse with certain movements. It would spontaneously subside to a dull ache before disappearing. She had had several evaluations and radiographic studies including ultrasound and contrast CT evaluations of her abdomen and pelvis, but without any definitive diagnosis. Notably, there was no clinical or radiographic evidence of inguinal or ventral hernias identified. She was otherwise healthy with only mild hypertension. She had had previous laparoscopic cholecystectomy and tubal ligation.

Her examination was unremarkable. Her vital signs were within normal limits; Body Mass Index (BMI) was 34.6. Specifically, the abdomen was tender in the right lower quadrant with no specific areas of point tenderness. There were no palpable hernias in the inguinal region or anywhere on the anterior abdominal wall. There were no discernible mass, although this was hampered by her truncal obesity body habitus. All surgical incisions were intact.

A repeat CT scan was ordered with oral and IV contrast and no abnormalities were found. Following a detailed discussion, a diagnostic laparoscopy was performed. She was found to have a right-sided Spigelian hernia measuring $2 \mathrm{~cm}$ and containing pre-peritoneal fat (Figure 1). A pre-peritoneal repair was performed using a light weight polypropylene mesh and absorbable tacks.

Following the procedure, she felt significantly improved and 8 months after surgery is still pain free.

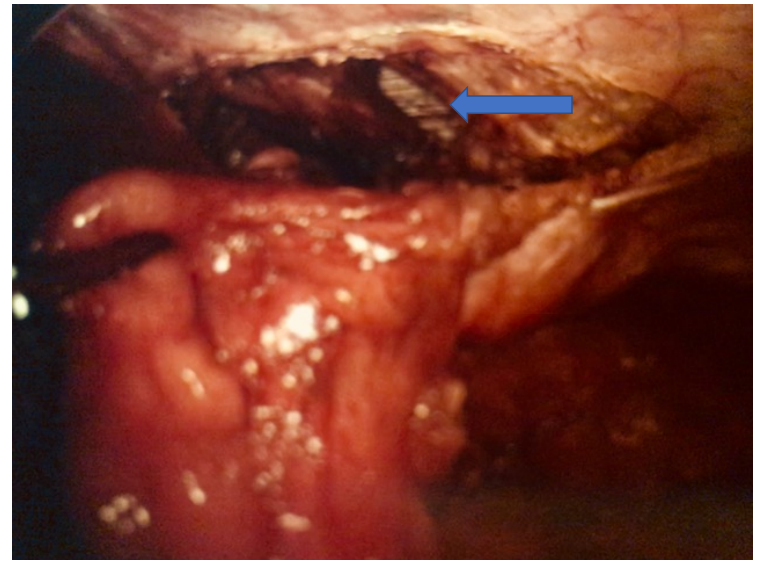

Figure 1. Spigelian hernia (arrow) following reduction of hernia contents

\section{Discussion}

The Spigelian hernia was named after the Flemish anatomist Adriaan van den Spieghel, who was the first person to describe the semilunar or Spigelian line. Another Flemish anatomist, Josef Klinkosch, would describe "Spigelian hernia" almost a century later [1]. It is a rare abdominal wall hernia that occurs at the inherently weak border of the semilunar line. They are most common below the arcuate line where the posterior sheath is absent. The hernia content usually contains preperitoneal fat from the plane just below the aponeuroses of the internal oblique muscle and the transversus muscle. The sac and its contents then lie below the level of aponeurosis of the external oblique. This characteristic can make diagnosis challenging.

${ }^{\star}$ Correspondence to: Shiva Seetahal, Department of Surgery, Heart of Florida Regional Medical Center (HOFRMC), Davenport, Florida, USA, Tel: 863-4217626; E-mail: shiva.seetahal@heartofflorida.com

Key words: spigelian hernia, Pre-peritoneal repair (TEP)

Received: June 01, 2018; Accepted: June 13, 2018; Published: June 15, 2018 
Classic clinical features include a lump or swelling in the lower abdomen along the semilunar line. This lump is most painful with extension movements of the trunk. Chronic pain is caused by pressure of the sac contents on the underlying nerves of the anterior abdominal wall that run along the internal oblique. This pain can be vague in nature with varying qualities. The clinical features of this hernia are so non-specific that less than half are diagnosed prior to surgical exploration [2-3].

The patient described in this case perfectly illustrates the diagnostic dilemmas that Spigelian hernias pose. The pain symptoms caused by the hernia can mimic a variety of other conditions including inguinal hernias, ureteric colic and pelvic organ pain. Furthermore, an obese abdomen impedes reliable palpation of the sac, even when clinically suspected. An abdominal ultrasound can detect the hernia with accuracy if the semilunar line is adequately scanned; this is driven by the physical findings in most cases. CT scanning is now ubiquitous for abdominal pain, and usually carries very high sensitivity and positive predictive value (PPV). It is very unusual that a focused scan was unable to detect the hernia, as in the case we described. MRI also offers nearperfect sensitivity for detection of these hernias but has a limited role due to cost and availability [3-4].

Repair of Spigelian hernias can be accomplished via open or laparoscopic approaches. Laparoscopically, the hernia can be closed with sutures and mesh can be placed extraperitoneally or intraperitoneally. They can also be repaired via a totally extraperitoneal approach ("TEP"). In this case, a Prolene mesh was placed in the preperitoneal space (Figure 2). This allows the advantage of covering the defect but shielding the bowel from contact with the mesh by repairing the peritoneal flap to sequestrate the mesh. All of these approaches carry excellent rates of success for non-obstructed hernias [5-7].

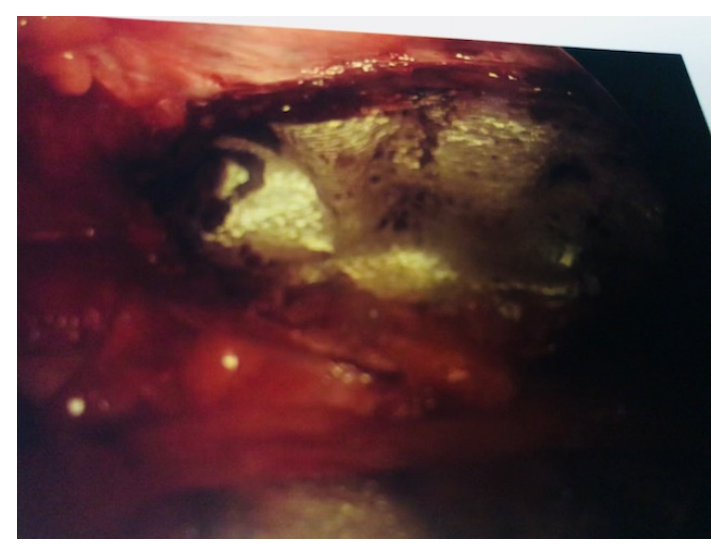

Figure 2. Mesh placed in preperitoneal space covering hernia defect.

\section{References}

1. Mittal T, Kumar V, Khullar R, Sharma A, Soni V, et al. (2008) [Not Available]. J Minim Access Surg 4: 95-98. [Crossref]

2. Pinna A, Cossu ML, Paliogiannis P, Ginesu GC, Fancellu A, et al. (2016) Spigelian hernia A series of cases and literature review. Ann Ital Chir 87: 306-311. [Crossref]

3. Larson DW, Farley DR (2002) Spigelian hernias: repair and outcome for 81 patients World J Surg 26: 1277-1281. [Crossref]

4. Campos SM, Walden T (1997) Images in clinical medicine. Spigelian hernia. $N$ Engl $J$ Med 336: 1149. [Crossref]

5. Majeski J (2009) Open and laparoscopic repair of Spigelian hernia. Int Surg 94: 365 369. [Crossref]

6. Yoshida D, Itoh S, Kinjo N, Harimoto N, Maruyama S, et al. (2015) Laparoscopic intraperitoneal mesh repair of Spigelian hernia: A case report. Asian J Endosc Surg 8: 477-479. [Crossref]

7. Moreno-Egea, Carrasco L, Girela E (2002) Open versus laparoscopic repair of spigelian hernia. A prospective randomized trial. Arch Surg 137: 1266-1268. [Crossref]

Copyright: $\odot 2018$ Seetahal S. This is an open-access article distributed under the terms of the Creative Commons Attribution License, which permits unrestricted use, distribution, and reproduction in any medium, provided the original author and source are credited. 